\title{
Fronto-orbito-zygomatic (FOZ) Approach
}

\author{
Imad N. Kanaan
}

\subsection{Introduction}

Surgical management of skull base pathologies remains one of the most challenging tasks for neurosurgeons. Advances in neuroimaging and evolution of modern technologies have paved the way for a more precise diagnosis and better selection of the surgical approach.

The fronto-orbito-zygomatic (FOZ) approach welds several surgical avenues and satisfies the philosophy of skull base surgery by removing bone obstacles in favor of better exposure with minimal brain retraction. Historically, this approach evolved from the pioneering work of avant-garde neurosurgeons. Removal of the supraorbital ridge as part of the frontal craniotomy was first described by McArthur [11] in 1912 and Frazier [5] in 1913 to remove a pituitary tumor. Jane et al. [8] revived this approach to include the anterior orbital roof osteotomy in a single flap to approach vascular lesions and remove orbital tumors. The original description of pterional approach was credited to Heuer [6] in 1918, modified by Dandy [3] to clip an anterior communicating artery aneurysm in 1941, and later was refined and popularized by Yasargil [14] in 1969. Pellerin [13] and Hakuba [7] first described

I. N. Kanaan

Department of Neurosciences,

King Faisal Specialist Hospital and Research Center, Alfaisal University, College of Medicine, Riyadh, Saudi Arabia the orbito-zygomatic-malar approach to central skull base lesions in the early 1980s. FOZ approach was eventually adopted and modified by Al Mefty [1] and others $[9,12,15]$ to include several variations that match the patient's clinical condition, the pathology dealt with, and the neurosurgeon's preference.

The FOZ approach provides an excellent neurosurgical avenue for safe removal of skull base tumors and management of complex vascular lesions around the central skull base, cavernous sinus, and upper clivus $[1,4,7,15]$. Several publications quantified this approach and highlighted its advantages in the contemporary practice of skull base surgery [2].

\subsection{Steps of the Approach}

\subsubsection{Position and Preparation}

The patient is placed in supine position with elevation of the trunk $25^{\circ}$ and ipsilateral shoulder (gravity-supported venous drainage). The head is elevated, extended, tilted slightly away from the ipsilateral shoulder, and rotated $25^{\circ}$ to the contralateral side (Fig. 3.1). The leg is prepped should a graft of fascia lata, fat, or saphenous vein be needed for reconstruction. Lumbar drain may be considered, and the patient receives antibiotics, steroids, H1-receptor antagonists, as well as anticonvulsant and mannitol if needed. 


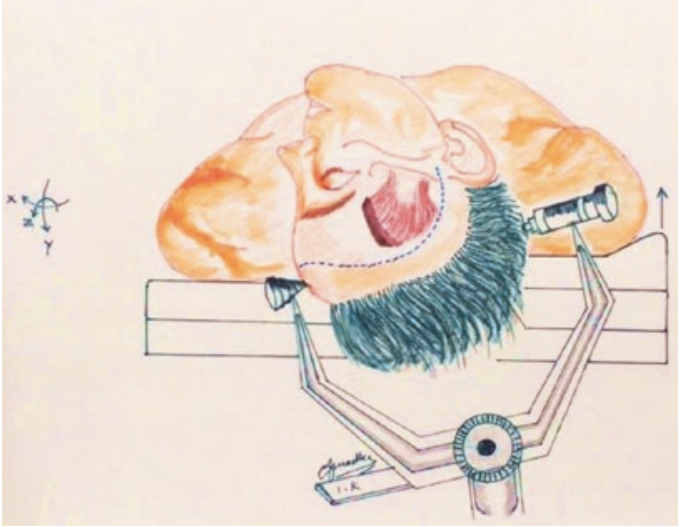

Fig. 3.1 Supine position with the head fixed in the Mayfield head clamp. The head and upper body is elevated up $25^{\circ}$ with elevation of the ipsilateral shoulder, hyperextended, and rotated to the opposite side by $25^{\circ}$. Additional rotation or manipulation of the position can be adjusted by the table's control buttons. The blue dotted line represents the skin incision. $X, Y, Z$ curved arrows represent the three axes of the head for orientation

\subsubsection{Skin Incision and Soft Tissue Dissection}

Elevate a frontal skin flap, starting within $1 \mathrm{~cm}$ anterior to the tragus at the level of the zygomatic arch, extending behind the hairline, and just passing the midline in a slightly curved fashion. Pay special attention not to injure the frontal branch of the facial nerve or damage the superficial temporal artery for its potential use in EC-IC bypass.

Incise and dissect sharply a large vascularized frontal pericranial flap and reflect it over the skin flap to be in continuity with the periorbita at the level of the orbital roof. Mobilize the temporal muscle basely and laterally under the arch of the zygoma, but leave a muscle cuff attached to the superior temporal line on the frontal bone for reattachment.

\subsubsection{Craniotomy and Orbito- zygomatic Osteotomy}

Perform two burr holes: one at McArthur's point (keyhole) and the second at the bottom of the

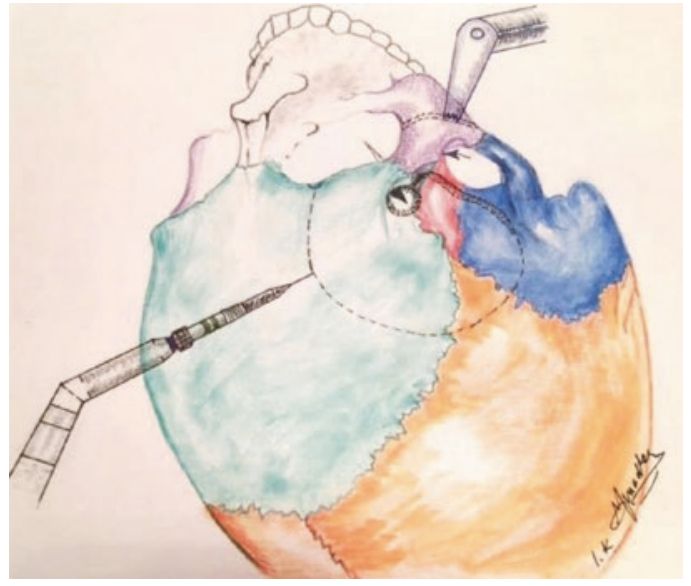

Fig. 3.2 Artistic illustration of the right fronto-orbitozygomatic approach (FOZ). Arrowhead, McArthur's burrhole; arrow, to inferior orbital fissure; dashed line, frontotemporal craniotomy including the orbital rim and part of the zygoma

temporal squama. Complete a frontotemporal craniotomy, and include the superior and lateral orbital rim (zygoma) medial to the supraorbital notch and down to the malar eminence (Fig. 3.2). This flap will include the anterior part of the orbital roof and the lateral wall of the orbit; hence, it negates the need for later reconstruction. We have used the oscillating saw and Midas Rex B1bone cutter or pediatric osteotome to perform this step. The cut of the orbital roof is placed lateral to the ethmoid sinuses, and the one at the lateral orbit is made between the outer edge of the superior and inferior orbital fissures. These cuts are best judged from an intraorbital perspective. Special attention is paid to dissect and protect both the frontobasal dura and the periorbita over the lacrimal gland using cotton patties or narrow brain spatula. This step can be performed as a single or two-piece bone flap. The latter is advocated in elderly patients with very thin dura or in patients with thick orbital roof. The elevation of this flap will facilitate the extradural resection of the sphenoid wing and drilling of the anterior clinoid process, as well as 
the decompression of the superior orbital fissure, the optic canal, and the posterior orbit using a high-speed diamond-tipped burr and small rongeurs. The need for the double cut of the zygomatic arc does not warrant total skeletonization of the bone and is limited to the extended subtemporal approach for surgical management of cavernous sinus lesions, complex basilar tip aneurysm, or upper clivus tumors. These cuts of the zygoma are now performed with pre-platting concept for best cosmetic results. For access to the cavernous sinus, petrous apex, and upper clivus, the lateral temporal fossa will be flattened using a high-speed drill; the foramen spinosum will be obliterated in order to proceed with extradural access to these areas as described by Dolenc [4] and Kawase [10], with special attention to maintain the integrity of the greater and lesser superficial petrosal nerves (GSPN and LSPN) during the exposure of the petrous carotid for proximal control (Fig. 3.3).

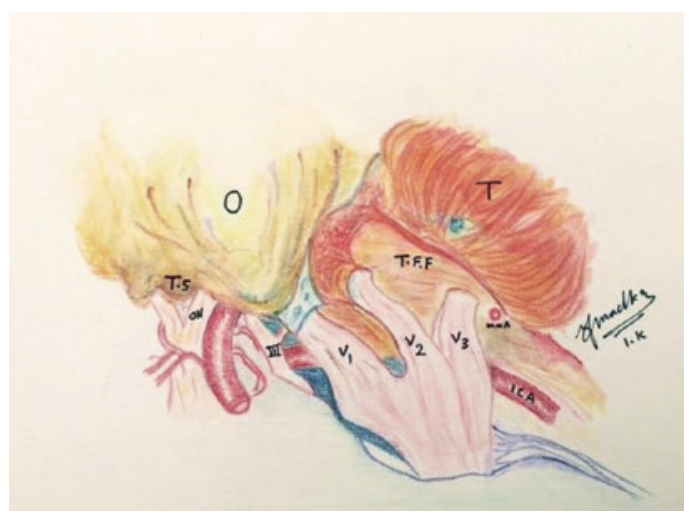

Fig. 3.3 Artistic illustration of a right front-orbitozygomatic (FOZ) approach. The wide exposure demonstrates access to the orbit, frontal fossa, central skull base, and suprasellar area, as well as to the cavernous sinus, middle fossa, and upper petroclival region. The following anatomical structures are shown: $T$ temporal muscle, $O$ orbit, $T F F$ temporal fossa floor, $T S$ tuberculum sellae, $O N$ optic nerve, ICA internal carotid artery, MMA middle meningeal artery, III oculomotor nerve, and $V 1, V 2, V 3$ represent the three branches of the trigeminal nerve

\subsubsection{Surgical Closure}

Upon conclusion of the planned intradural intervention, the dura is closed in a watertight fashion using dura substitutes in order to guarantee brain relaxation and tent the dura to prevent postoperative epidural collection. The fronto-orbitozygomatic bone flap is replaced and fixed in position with miniplates. The preservation of the roof and lateral wall of the orbit does not warrant additional bone reconstruction; however, if the osteotomy defect is large, then this can be reconstructed by using low-profile craniofacial miniplates that can be fixed to the FOZ flap and measured to the size and shape of the bone defect. The temporal muscle and fascia flap are sutured to the myofascial cuff left on the frontal bone. The skin incision is closed in a multilayer fashion leaving a small subgaleal closed drainage system.

\subsection{Indications of the Approach}

Fronto-orbito-zygomatic approach provides multiple short, wide, and direct corridors giving access for safe surgical management of complex skull base tumors and vascular pathologies located in the posterior orbit or at the central skull base, interpeduncular fossa, cavernous sinus, and upper clivus.

Variations of FOZ approach [1, 7-9, 12, 15] are tailored to the nature of the pathology being dealt with and its anatomical location and extent and governed by meticulous evaluation of the patient's clinical condition and imaging studies. The FOZ approach or its variants (Figs. 3.4, 3.5, and 3.6) are to be considered in surgical management of suprasellar, perisellar, and anterior clinoid meningiomas, craniopharyngiomas, giant pituitary tumors, trigeminal schwannomas, chordomas, cavernous sinus lesions, and complex anterior circulation, or basilar bifurcation aneurysms. A trans-eyebrow mini-FOZ approach was described in the literature and may suffice adequately in some selected indications [9]. 

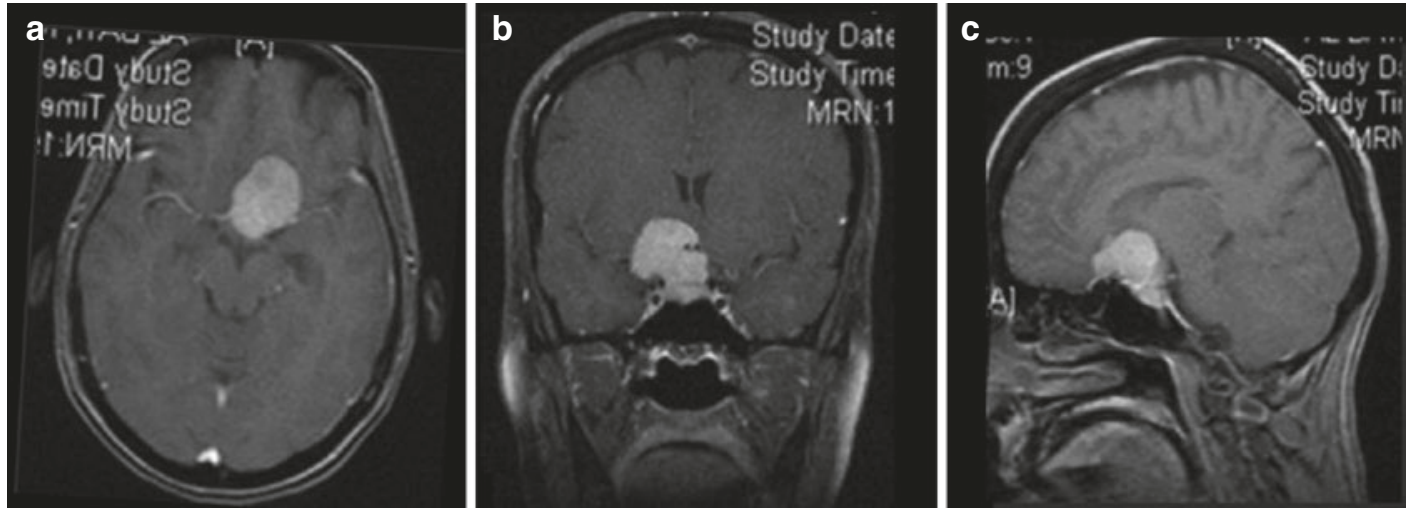

Fig. 3.4 Preoperative MRI T1 with gadolinium of a 42-year-old female patient who presented with 8 months' history of headache and progressive visual impairment, more pronounced on the right side. Her neurological examination confirmed the presence of right optic nerve atrophy, decreased visual acuity to 20/100, and temporal visual field defect; (a) axial; (b) coronal; (c) sagittal projection showing homogenous enhancement of the lesion
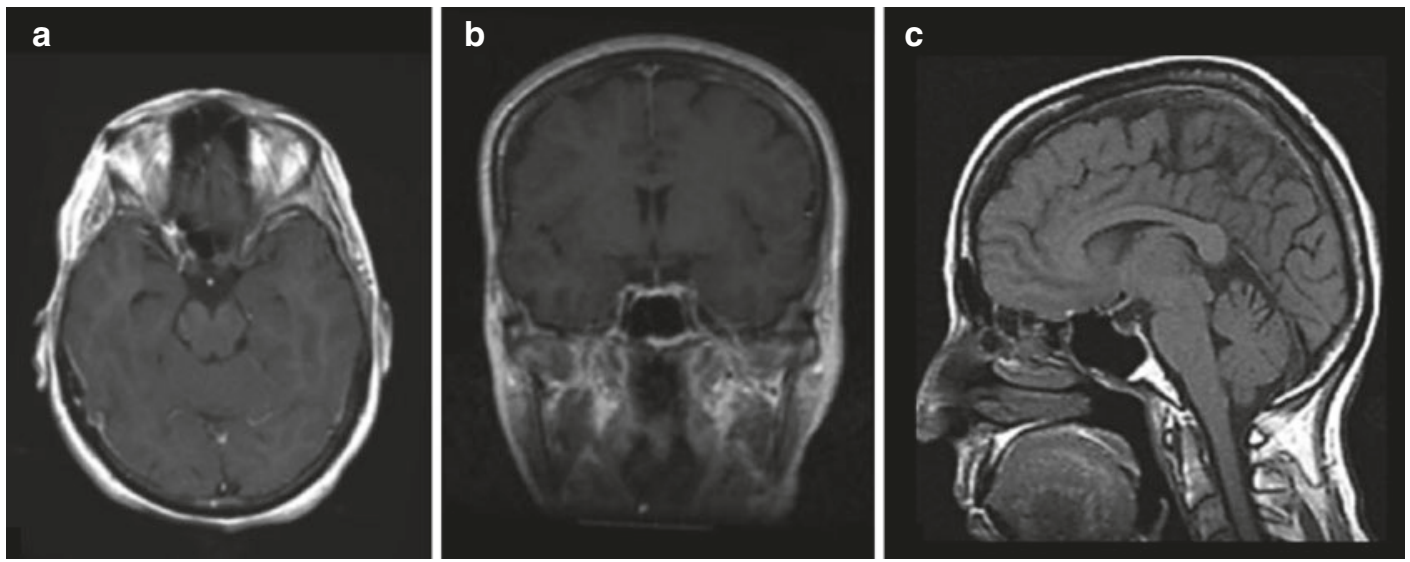

Fig. 3.5 Postoperative MRI with gadolinium; (a) axial; (b) coronal; (c) sagittal views. It demonstrates complete resection of the diaphragma sellae meningioma. The patient underwent a modified FOZ approach with opening of the optic canal and resection of the sphenoid wings and sparing the cut of the zygoma arc. The patient reported a full recovery of her vision and symptoms at the 9-month follow-up with no complications

\subsection{Limitation and Complication and How to Avoid}

The FOZ approach may appear to be a complex intervention, but it is worth the effort whenever it is indicated. It provides excellent working channels and maneuverability to handle intraoperative complications. Risks of facial nerve injury and temporal muscle atrophy can be avoided by the subperiosteal elevation of the temporal muscle and minimal use of cautery. The approach as described above negates the need for major reconstruction; however, enophthalmos may occur in some cases as the result of greater bone loss due to excessive drilling or damage to the periorbital area during dissection. Therefore, proper placement of the osteotomy at the orbital roof and lateral wall under protection of the periorbita and repair of any ensuing defect in the periorbita by using a small pericranial patch are very important measures. It is recommended to reconstruct a larger bone defect in the orbita by 

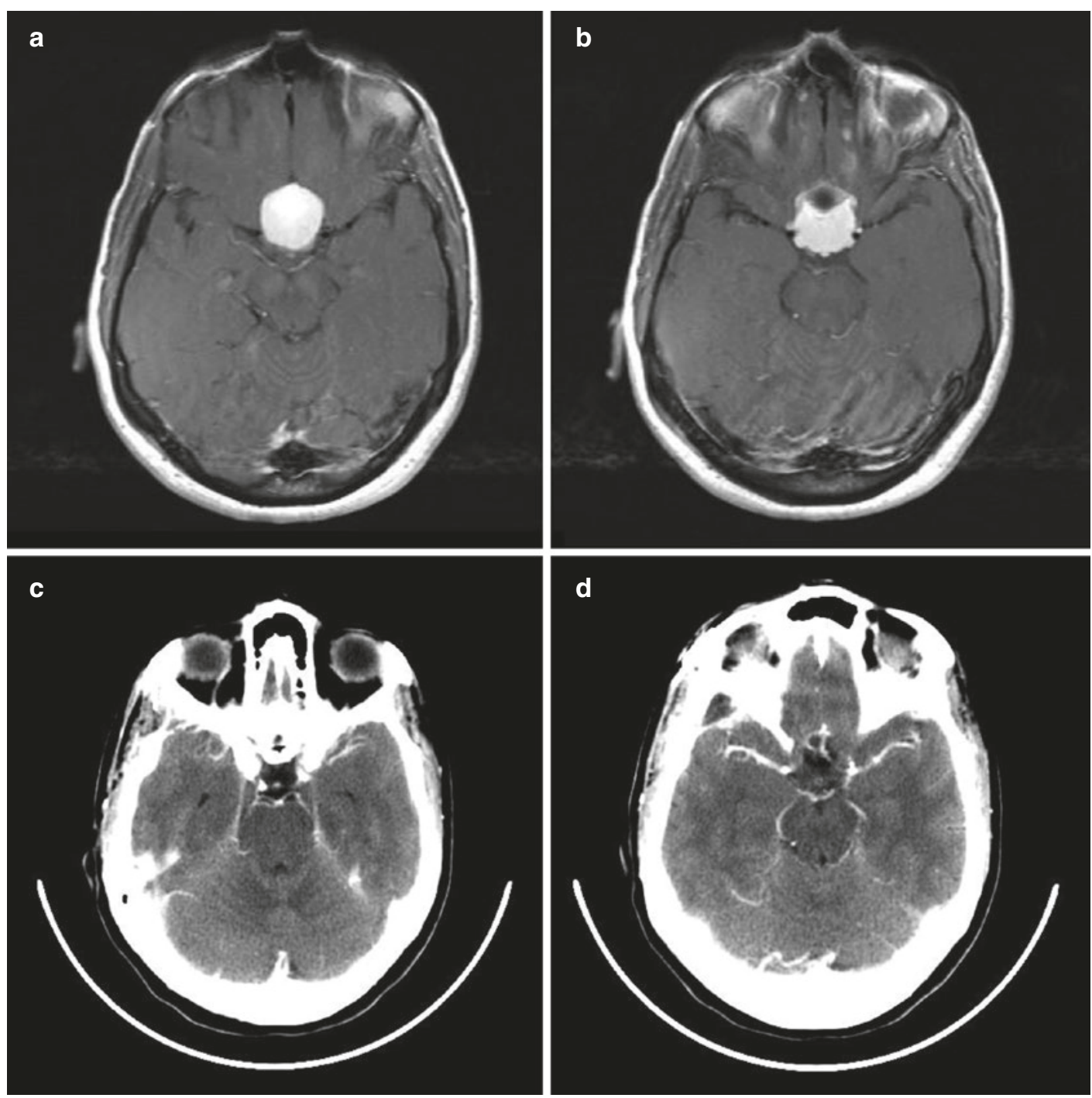

Fig. 3.6 Preoperative MRI, T1 with gadolinium (a and b) of a middle-age female patient, who presented with 1-year history of progressive visual loss and recent episode of generalized seizure. These images revealed a planum sphenoidale lesion with hyperostosis, which was compat-

ible with the diagnosis of meningioma. The patient underwent trans-eyebrow modified mini-FOZ for gross total resection of the planum sphenoidale meningioma as shown on the postoperative CT scan with contrast (c and d) resulting an excellent clinical recovery

using miniplates that can be fixed to the bone flap. Careful repair and obliteration of any opened air cells or air sinuses should handle the potential risk of CSF leakage resulting from drilling of the anterior clinoid process or the wall of the sinuses. The relatively common problem of transient periorbital swelling can be managed by early ambulation, head elevation, and application of cold compress.

\section{References}

1. Al-Mefty O. Supraorbital-pterional approach to skull base lesions. Neurosurgery. 1987;21:474-7.

2. Andaluz N, Keller JT, van Loveren HR, Zuccarello M. Anatomical and clinical study of the orbitopterional approach to anterior communicating artery aneurysms. Neurosurgery. 2003;52(5):1140-9.

3. Dandy WE. Intracranial arterial aneurysms. New York: Hafner; 1944 Reprinted 1969. 
4. Dolenc VV. Extradural approach to intracavernous ICA aneurysms. Acta Neurochir Suppl. 1999;72:99-106.

5. Frazer $\mathrm{CH}$. An approach to the hypophysis through the anterior cranial fossa. Ann Surg. 1913;57(2):145-50.

6. Heuer GJ, Dandy WE. A new hypophysis operation. Johns Hopkins Hosp Bull. 1918;29:154.

7. Hakuba A, Liu SS, Shuro N. The orbitozygomatic infratemporal approach: a new surgical technique. Surg Neurol. 1986;26(3):271-6.

8. Jane JA, Park TS, Pobereskin LH, et al. The supraorbital approach: technical note. Neurosurgery. 1982;11(4):537-42.

9. Kanaan IN. Trans-eyebrow mini-orbitozygomatic pterional approach for minimally invasive skull base surgery. Minim Invas Neurosurg. 2005;48(1):34-8.

10. Kawase T, Toya S, Shiobara R, et al. Transpetrosal approach for aneurysms of the lower basilar artery. J Neurosurg. 1985;63:857-61.
11. McArthur LL. An aseptic surgical access to the pituitary body and its neighborhood. JAMA. 1912;58:2009-11.

12. McDermott MW, Durity FA, Rootman J, et al. Combined frontotemporal-orbitozygomatic approach for tumors of the sphenoid wing and orbit. Neurosurgery. 1990;26(1):107-16.

13. Pellerin P, Lesoin F, Dhellemmes P, et al. Usefulness of the orbitofrontomalar approach associated with bone reconstruction for frontotemporosphenoid meningiomas. Neurosurgery. 1984;15(5):715-8.

14. Yasargil MG, Antic J, Laciga R, Jain KK, Hodosh RM, Smith RD. Microsurgical pterional approach to aneurysm of the basilar bifurcation. Surg Neurol. 1976;6(2):83-91.

15. Zabramski JM, Kiris T, Sankhla SK, Cabiol J, Spetzler RF. Orbitozygomatic craniotomy: Technical note. J Neurosurg. 1998;89(2):336-41.

Open Access This chapter is licensed under the terms of the Creative Commons Attribution 4.0 International License (http://creativecommons.org/licenses/by/4.0/), which permits use, sharing, adaptation, distribution and reproduction in any medium or format, as long as you give appropriate credit to the original author(s) and the source, provide a link to the Creative Commons license and indicate if changes were made.

The images or other third party material in this chapter are included in the chapter's Creative Commons license, unless indicated otherwise in a credit line to the material. If material is not included in the chapter's Creative Commons license and your intended use is not permitted by statutory regulation or exceeds the permitted use, you will need to obtain permission directly from the copyright holder. 